\title{
Sueño y Estrés: Su Relación Con el Rendimiento Académico En Niños
}

Iglesia, Fabiola ${ }^{1\left({ }^{*}\right), 2 \text { y }}$; Lopez, Magdalena ${ }^{1}$; Rosler, Roberto ${ }^{2}$; Logatt Grabner, Carlos ${ }^{3}$; Logatt Grabner, Cristian ${ }^{3}$; Castro, María ${ }^{3}$

${ }^{1}$ Centro de Investigación Interdisciplinar en Valores, Integración y Desarrollo Social (CIIVIDS).

${ }^{2}$ Pontificia Universidad Católica Argentina (UCA). Buenos Aires, Argentina.

${ }^{3}$ Asociación Educar para el Desarrollo Humano. Buenos Aires, Argentina

\section{RESUMEN}

Introducción: una buena calidad y cantidad de sueño nocturno es un factor esencial para permitir un adecuado funcionamiento diurno. El sueño puede alterarse debido a una multiplicidad de factores, reconociendo al estrés experimentado durante el día como uno de ellos. Este trabajo propone analizar en qué medida el estrés percibido y la calidad y cantidad de sueño afectan el desempeño académico de escolares de educación primaria. Metodología: La muestra estuvo conformada por 465 estudiantes de 6 a 12 años de edad, y sus docentes, quienes respondieron preguntas sobre rendimiento académico, calificándolo según una escala de 5 puntos. Los niños respondieron el Inventario de Estrés Cotidiano Infantil y el Cuestionario BEARS para conocer la calidad y cantidad de sueño. Resultados: Encontramos que existe una deuda de sueño en todas las edades, El 54,6\% de niños no duermen las horas recomendadas y un $83,4 \%$ reportan diferentes dificultades de sueño. El 48,4 \% de niños muestran un alto nivel de estrés. Los niños que manifiestan menor percepción de estrés y mejor perfil de sueño son los que obtienen un mejor rendimiento académico. Conclusiones: Los hallazgos exponen la necesidad de un abordaje psicoeducativo de estas variables para optimizar los desempeños escolares y promover la salud en esta etapa evolutiva.

Palabras clave: Calidad de sueño. Cantidad de sueño. Estrés. Niños. Rendimiento académico.

\section{Sleep and Stress: Its Relationship with School Performance in Children}

\section{ABSTRACT}

Introduction: A good quality and quantity of night-time sleep is an essential factor in enabling adequate daytime functioning. Sleep can be altered due to a multiplicity of factors, acknowledging stress experienced during the day as one of them. This study proposes to analyze to what extent perceived stress and the quality and quantity of sleep affect the academic performance of elementary school students. Methodology: The sample consisted of 465 students from 6 to 12 years old and their teachers, who answered questions about academic performance, rating it according to a 5-point scale. Children answered the Children's Daily Stress Inventory (Inventario Infantil de Estresores Cotidianos, IIEC) and the BEARS Questionnaire in order to know the quality and quantity of sleep. Results: We found out that there is a sleep debt in all ages. 54,6 \% of children do not sleep the recommended hours and $83,4 \%$ reported different sleep difficulties. $48,4 \%$ of children show a high level of stress. Children who show lower stress perception and a better sleep profile are the ones who obtain better academic performance. Conclusions: These findings set out the need for a psychoeducational approach to these variables in order to optimize school performances and promote health at this evolutionary stage.

Keywords: Quality of sleep. Amount of sleep. Stress. Children Academic performance.

Recibido: 24/05/2021 Aceptado:01/06/2021

Correspondencia: (*) fabiolaiglesia@uca.edu.ar 


\section{INTRODUCCIÓN}

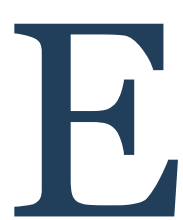

$\mathrm{n}$ los últimos años, se ha observado un descenso en la duración y calidad promedio del sueño en la población mundial, con consecuencias negativas para la salud en general.

Una buena calidad y cantidad de sueño nocturno es un factor fundamental para permitir un adecuado funcionamiento durante el día.

Numerosas investigaciones muestran una reducción en la cantidad de horas de sueño, incluso en la población infantil. Afectando, la restricción de horas de sueño, el estado de alerta y las tareas que requieren control ejecutivo (Fallone, et al., 2001).

Por otro lado, durante la etapa de la niñez, el estrés cotidiano es un factor que impacta negativamente sobre el aprendizaje y desarrollo (González et al. 2014).

Uno de los factores que modifica el sueño es el estrés experimentado durante el día y esta condición puede afectar la cantidad de sueño, su calidad, o ambas.

La relación entre el sueño y el estrés se puede comprender como una doble vía, debido a que las dificultades en el sueño podrían ocasionar estrés y este a su vez provocaría alteraciones en el ciclo de sueño (Del Río Portilla, 2006).

\section{MARCO TEÓRICO}

El sueño puede definirse como un estado de quietud conductual, acompañado de inmovilidad y una disminución en la capacidad para responder a estímulos externos (Astrobus, et al., 1993).

Muchos avances en el conocimiento sobre el sueño se han realizado en los últimos años.

Sabemos que el sueño es activado y desactivado como resultado de una gama de interacciones, controlado por la melatonina, una hormona que se encarga de avisar al organismo del momento del día en que se encuentra, generando los ritmos circadianos que indican cuándo es necesario dormir y cuándo estar despierto. El ritmo circadiano es regulado por unas células que detectan la presencia de luz, que inhibe la melatonina, indicando que es de día, mientras que la oscuridad desencadena los mecanismos cerebrales necesarios para el sueño al elevarse los niveles de esta hormona.

En el sueño existen al menos dos etapas, el sueño lento (SL y sus etapas) y el sueño de movimientos oculares rápidos (MOR), que alternan en una noche de sueño varias veces, constituyendo un ritmo intrínseco ultradiano. En los seres humanos adultos el sueño nocturno se caracteriza por 4 a 6 ciclos ultradianos. Se entiende por ciclo ultradiano aquel período que se inicia al empezar el SL y termina al finalizar una etapa de MOR, repitiéndose varias veces en la noche. Aunque la duración de cada ciclo ultradiano es variable, el promedio es de aproximadamente 90 minutos (Velluti y Pedemonte, 2006).

Las etapas del sueño cambian con la edad. Después de los 5 años de edad los patrones de sueño se mantienen relativamente constantes, en condiciones normativas, durante el resto de la vida (Shellhaas et al, 2017; Feinberg, et al., 2010).

En la actualidad, la evidencia científica propone que para conseguir un óptimo estado de alerta durante el día, durante la etapa de 5 a 10 años, los niños duerman entre 9 y 10 horas durante la noche (Biggs, et al., 2011; Brand, et al., 2010).

Pese a esta información, hay estudios que revelan que el $45 \%$ o más de los niños duermen menos de 8 horas diarias (Dewald et al., 2010; Rosler et al., 2019).

Además de la disminución en las horas de sueño, postergar la hora de acostarse y levantarse temprano también afecta la capacidad de aprendizaje de los alumnos, su funcionamiento neurocomportamental, y consecuentemente, su rendimiento académico (Cladellas Pros, et al. 2015; Fallone, et al., 2006; Khalil y Salah, 2012; Lopez y Iglesia, 2019a).

Diferentes trabajos han explicado que cuando los niños postergan la hora de acostarse luego de las 22 horas les resulta más difícil poder levantarse temprano al día siguiente (Yokomaku et al., 2008); el demorar la hora de acostarse, en niños de edad escolar, disminuye la cantidad de horas de sueño (Rosler, et 
al., 2019).

Esta disminución en la cantidad de horas de sueño y la postergación en la hora de acostarse tendrían efectos negativos en el rendimiento académico (BaHamman, et al., 2006; Lopez y Iglesia, 2019a).

Por otra parte, sabemos que el estrés se genera cuando determinados eventos de la vida, ya sean de orden físico o psíquico, superan los recursos para afrontarlos.

Selye (1956), define el estrés como un conjunto de respuestas fisiológicas inespecíficas a estímulos físicos externos o internos. Explicitando tres etapas en la respuesta al estrés, en la primera etapa, alarma, el cuerpo reconoce la amenaza que y se prepara para la acción, ya sea de agresión o de fuga (Selye, 1946). En la resistencia, segunda etapa, el organismo se repara el daño causado por la reacción de alarma y si el estrés continúa, permanece la alerta y no pueden repararse los daños. Si continúa la resistencia, comienza el agotamiento, la tercera etapa, cuya resultado puede ser ya una alteración producida por el estrés crónico. La exposición prolongada al estrés agota las reservas de energía del cuerpo y puede llevar a situaciones extremas.

Por su parte Lazarus refiere que el estrés surge a partir de la relación establecida entre la persona con su ambiente, provocando importantes modificaciones en su percepción de bienestar (Lazarus and Folkman, 1984).

Atendiendo a la concepción de estrés como estímulo, los estresores se clasifican en sucesos vitales, crónicos y cotidianos (Sandín, 2003).

El estrés diario puede definirse como los constantes acontecimientos negativos que ocurren como consecuencia de la interacción de diferentes factores de la realidad que vive una persona con su entorno o circunstancias en las que vive (Kanner, et al. 1981), considerándose como estresores de vida diarios a todos aquellos acontecimientos que tiene una elevada probabilidad de ocurrir y reiterarse de manera continua, ocasionando consecuencias relevantes en la vida cotidiana, debido a su frecuencia. Presentan baja intensidad y alta frecuencia (Seiffge-Krenke,
2000). A su vez, el estrés diario o cotidiano puede tener un impacto más negativo aun en el desarrollo emocional del niño o adolescente que el estrés debido a sufrir acontecimientos vitales o estresores crónicos (Seiffg-Krenke, 2000; Wagner, Compas and Howell, 1988).

Por este motivo es que están surgiendo cada vez más estudios que muestran que los niveles de estrés en los niños han aumentado en las últimas décadas (Maturana y Vargas, 2015). El desarrollo socioemocional de los niños se ve influido por las demandas que se producen en la interacción diaria con su entorno.

En población escolar, los estresores de naturaleza cotidiana se agrupan principalmente en tres ámbitos: salud, escuela y familia (Trianes, et al., 2011). Estudios previos, en niños de diferentes edades, muestran asociaciones entre altos niveles de estrés cotidiano y ansiedad, baja autoestima, depresión y sentimientos de incapacidad (Trianes, et al., 2012). Se han observado además, asociaciones con dificultades en las relaciones interpersonales con pares, irritabilidad, conductas agresivas y actitud negativa hacia el ámbito escolar (Trianes, et al., 2014) y una disminución en el rendimiento académico (Martinez, et al., 2019; Lopez y Iglesias, 2019b; Valiente-Barroso, et al., 2020).

El rendimiento académico se ve afectado por una variedad de factores que pueden influir en los procesos de aprendizaje del alumnado en edades tempranas del desarrollo. Debido a la importancia que tienen estas variables para el desarrollo integral de niños es que este trabajo se propuso conocer la relación con el rendimiento académico de las dificultades en la calidad y cantidad de sueño y los altos niveles de estrés percibido en población infantil. 


\section{METODOLOGÍA}

\subsection{Diseño de estudio}

Se realizó un estudio de tipo ex post facto de corte transversal, descriptivo y correlacional.

\subsection{Muestra}

Se trabajó con una muestra intencional, no probabilística de 465 estudiantes con edades comprendidas entre los 6 y 12 años de edad (46,7\% mujeres y 53,3 hombres, con una media de edad de $M=8,46 ; \mathrm{DE}=$ $1,77)$ y sus respectivos docentes, residentes en diferentes provincias de Argentina que cursaban la escolaridad primaria en escuelas de gestión pública y privada. El criterio de inclusión fue que los niños no presentaran antecedentes clínicos, neurológicos ni psiquiátricos y que cursasen sus estudios escolares con regularidad, sin repitencia escolar (ver Tabla 1).

Tabla 1. Características sociodemográ ficas de la muestra

\begin{tabular}{ccccc}
\hline Género & 6 a 8 años & 9 a 10 años & $\mathbf{1 1}$ a 12 años & Total \\
\hline Femenino & 125 & 67 & 25 & 217 \\
Masculino & 122 & 74 & 52 & 248 \\
Total & 247 & 141 & 77 & 465 \\
\hline
\end{tabular}

\subsection{Instrumentos}

Los docentes respondieron a preguntas sobre rendimiento académico. Los docentes calificaron a los alumnos según una escala de 5 puntos: 1 . Muy por debajo de la media de la clase 2. Por debajo de la media de la clase 3. En la media de la clase 4 . Por encima de la media de la clase 5 . Muy por encima de la media de la clase.

Los niños respondieron:

El Inventario de Estrés Cotidiano Infantil IECI (Trianes, et al., 2009), que consta de 22 ítems dicotómi$\cos (\mathrm{Si} / \mathrm{No})$, que describen la ocurrencia de diversos sucesos, problemas, contrariedades y preocupaciones que se originan en la interacción diaria con el entorno, susceptibles de una reacción emocional y que pueden afectar de forma negativa al desarrollo de los niños en edad escolar. El instrumento proporciona una puntuación total de estrés cotidiano en niños de 6 a 12 años y puntuaciones parciales del estrés en problemas de salud y psicosomáticos (8 ítems), en el ámbito escolar (7 ítems), en el ámbito familiar (7 ítems).

El Cuestionario BEARS (Owens y Dalzell, 2005) indicado para conocer la calidad y cantidad de sueño de los niños y adolescentes de 2-18 años de edad. Valora cinco aspectos del sueño: existencia de problemas al acostarse, somnolencia diurna excesiva, despertares nocturnos, regularidad y duración del sueño y presencia de ronquido. Establece tres grupos de edad: 2 a 5 años, 6 a 12 años y de13 a 18 años, con preguntas dirigidas a los niños y a sus padres.

\subsection{Procedimiento de recolección de datos}

Se solicitó la autorización de los directivos de las escuelas con quienes se tuvo una primera entrevista informativa del estudio a realizar y se solicitó informara a los docentes de los grados con quienes se trabajaría. Posteriormente, se informó a los padres y se requirió el consentimiento informado como requisito para la participación de sus hijos.

A los estudiantes se les comunicó las características de las tareas por realizar, luego se los invitó a participar y se les informaron sus derechos como participantes y que la participación era voluntaria, luego, una vez obtenido el consentimiento y asentimiento de cada participante se completaron los instrumentos de manera individual.

Por otra parte, se solicitó a los docentes de los niños participantes que respondieran el cuestionario sobre Rendimiento Académico y a los padres o tutores de los niños participantes que respondieran las preguntas del BEARS que requerían su participación.

\subsection{Análisis de datos}

El procesamiento y análisis estadístico de los datos, se llevó a cabo utilizando el Statistical Package for the Social Sciences (SPSS) versión 23.0.

Se calcularon porcentajes y medias para describir la cantidad, calidad de sueño y estrés reportado por los niños. Para analizar la relación entre el rendimiento académico según el perfil de sueño y la percepción de estrés, se empleó Análisis Multivariado de Varianza (MANOVA). Para comprobar el tamaño del efecto (effect size) de las Fs multivariadas y univariadas se emplearon Eta parciales al cuadrado. 


\section{RESULTADOS Y/O DISCUSIÓN}

Los resultados de los estadísticos descriptivos de las variables incluidas en este estudio aparecen en la Tabla 2 e indican las frecuencias y porcentajes de aparición en cada grupo de edad estudiado.

Tabla 2. Características del sueño según la edad.

\begin{tabular}{|c|c|c|c|c|c|c|c|c|c|}
\hline \multicolumn{2}{|c|}{ Edad } & \multicolumn{2}{|c|}{6 a 8} & \multicolumn{2}{|c|}{9 a 10} & \multicolumn{2}{|c|}{11 a 12} & \multicolumn{2}{|c|}{ Total } \\
\hline & & $\mathbf{n}$ & $\%$ & n & $\%$ & n & $\%$ & $\mathbf{n}$ & $\%$ \\
\hline \multirow{3}{*}{$\begin{array}{l}\text { Horas de } \\
\text { Sueño }\end{array}$} & Menos de 9 hs. & 130 & 52,6 & 77 & 54,6 & 47 & 61 & 254 & 54,6 \\
\hline & 9 a 10 hs. & 83 & 33,6 & 48 & 34 & 20 & 26 & 151 & 32,5 \\
\hline & Más de 10 hs. & 34 & 13,8 & 16 & 11,3 & 10 & 13 & 60 & 12,9 \\
\hline \multirow{2}{*}{$\begin{array}{l}\text { Hora de } \\
\text { Dormir }\end{array}$} & Antes de 22 hs. & 119 & 48,2 & 63 & 44,7 & 27 & 35,1 & 209 & 44,9 \\
\hline & Después de 22 & 128 & 51,8 & 78 & 55,3 & 50 & 64,9 & 256 & 55,1 \\
\hline \multirow{2}{*}{$\begin{array}{l}\text { Dificultades } \\
\text { de Sueño }\end{array}$} & No reporta & 38 & 15,4 & 24 & 17 & 15 & 19,5 & 77 & 16,6 \\
\hline & Reporta & 209 & 84,6 & 141 & 83 & 77 & 80,5 & 338 & 83,4 \\
\hline
\end{tabular}

Como muestran los resultados el porcentaje de niños que duerme menos de las horas recomendadas para su edad se incrementa a medida que aumenta la edad.

Si comparamos la cantidad de horas de sueño recomendadas y la informada por los niños se observa una diferencia negativa, el $54,6 \%$ de los niños no duermen las horas recomendadas.

Como puede verse existe una deuda de sueño en todas las edades evaluadas.

Si se analiza el horario de acostarse en la muestra total se observa que más de la mitad de los niños de primaria demoran la hora de acostarse y el $80 \%$ refiere tener alguna dificultad relacionada a la calidad del sueño.

Tabla 3. Percepción de estrés según la edad.

\begin{tabular}{cccccccccc}
\hline \multicolumn{1}{c}{} & \multicolumn{2}{c}{$\mathbf{6} \mathbf{a} \mathbf{8}$} & \multicolumn{2}{c}{$\mathbf{9} \mathbf{a} \mathbf{1 0}$} & \multicolumn{11}{c}{$\mathbf{1 1}$} & \multicolumn{2}{c}{ Total } \\
\hline & & $\mathbf{n}$ & $\mathbf{\%}$ & $\mathbf{n}$ & $\mathbf{\%}$ & $\mathbf{n}$ & $\mathbf{\%}$ & $\mathbf{n}$ & $\mathbf{\%}$ \\
Estrés & Estrés Alto & 121 & 49 & 72 & 51,1 & 47 & 61 & 240 & 51,6 \\
Total & Estrés Bajo & 126 & 51 & 69 & 48,9 & 30 & 39 & 225 & 48,4 \\
\hline & & & & & & & & & \\
Estrés & Estrés Alto & 117 & 47,4 & 51 & 36,2 & 18 & 23,4 & 186 & 40 \\
Salud & Estrés Bajo & 130 & 52,6 & 90 & 63,8 & 59 & 76,6 & 279 & 60 \\
\hline & & & & & & & & & \\
Estrés & Estrés Alto & 114 & 46,2 & 76 & 53,9 & 34 & 44,2 & 224 & 48,2 \\
Escuela & Estrés Bajo & 133 & 53,8 & 65 & 46,1 & 43 & 55,8 & 241 & 51,8 \\
\hline & & & & & & & & & \\
Estrés & Estrés Alto & 159 & 64,4 & 64 & 45,4 & 37 & 48,1 & 260 & 55,9 \\
Familia & Estrés Bajo & 88 & 35,6 & 77 & 54,6 & 40 & 51,9 & 205 & 44,1 \\
\hline
\end{tabular}

La variable de estrés se categorizó en dos niveles: alto y bajo. Para lo cual se utilizó la media estadística obtenida por la muestra total y en cada componente, considerando a los estudiantes con un nivel alto de estrés a quienes puntúan por encima de la media de la muestra y con un nivel bajo de estrés aquellos que quedan por debajo de la media estadística.

En la Tabla 3 puede observarse que al consultar a los niños sobre la percepción de estrés encontramos que el $51,6 \%$ manifiesta en sus respuestas niveles altos de preocupaciones o estrés.

Cuando analizamos la composición del estrés, en las tres dimensiones, encontramos que las preocupaciones varían según la edad, los niños más pequeños reportan mayor nivel de preocupaciones que pertenecen al ámbito familiar, seguidas de salud y por último las escolares.

Considerando el grupo etario en general las preocupaciones más altas pertenecen al ámbito familiar, seguidas del escolar y por último salud.

El Rendimiento Académico se categorizó en dos niveles; por debajo de la media (RA Bajo, $n=229$ ), y por encima de la media (RA Alto).

Para analizar las diferencias en el Rendimiento Académico según el nivel de estrés y el perfil de sueño se empleó MANOVA. Los resultados indican que existen diferencias significativas según el Rendimiento Académico, el perfil de sueño y el nivel de estrés, $F$ de Hotelling $(6,458)=9,590 p=.000, \eta 2$ parcial $=.0,112$.

Al analizar los $\mathrm{F}$ univariados se observan diferencias significativas en cuanto a las horas de sueño $F(1,463)$ $=6,174 p=.013, \eta 2$ parcial $=.0,013$, en cuanto a la hora de acostarse $F(1,463)=5,965 p=.015, \eta 2$ parcial $=.0,013$, y en cuanto a las dificultades de sueño $F(1,463)=6,121 p=.014, \eta 2$ parcial $=.0,013$ (Ver Tabla 4).

De manera que los estudiantes con un mejor perfil de sueño tienen un mejor puntaje en rendimiento académico.

Se observan diferencias significativas en estrés total $F(1,463)=41,950 p=.000, \eta 2$ parcial $=.0,083$, y en las dimensiones de salud $F(1,463)=15,608 p=.000$, $\eta 2$ parcial $=$. 0,033, escolar $F(1,463)=41,771 p=$ $.000, \eta 2$ parcial $=.0,083$ y familiar $F(1,463)=20,919$ $p=.000, \eta 2$ parcial $=.0,043$. 
Tabla 4. Perfil de Sueño, Estrés y Rendimiento Académico.

\begin{tabular}{|c|c|c|c|c|c|c|c|}
\hline & \multicolumn{2}{|c|}{ RA Bajo } & \multicolumn{2}{|c|}{ RA Alto } & \multirow{2}{*}{$\begin{array}{c}F \\
(1,463)\end{array}$} & \multirow[t]{2}{*}{$p$} & \multirow{2}{*}{$\begin{array}{c}\text { Eta }^{2} \\
\text { parcia }\end{array}$} \\
\hline & M & $\overline{D E}$ & $\mathbf{M}$ & $\mathbf{D E}$ & & & \\
\hline Horas de Sueño & 8,36 & 1,74 & 8,74 & 1,50 & 6,174 &, 013 & , 013 \\
\hline Hora de Dormir & 22,95 & 1,40 & 22,64 & 1,33 & 5,965 & 015 & 013 \\
\hline $\begin{array}{l}\text { Dificultades de } \\
\text { Sueño }\end{array}$ & 1,93 & 1,19 & 1,65 & 1,24 & 6,121 & ,014 & ,013 \\
\hline Estrés Total & 8,54 & 3,45 & 6,48 & 3,39 & 41,950 & ,000 & ,083 \\
\hline Estr & 0,42 & 0,21 & 0,34 & 0,22 & 15,608 & ,000 & 033 \\
\hline Estrés Escuela & 0,42 & 0,20 & 0,30 & 0,18 & 41,771 & ,000 &, 083 \\
\hline Estrés Familia & 0,31 & 0,21 & 0,23 & 0,19 & 20,919 & , 000 & 043 \\
\hline
\end{tabular}

Nota: $M=$ media aritmética. $D E=$ desvío estándar. $\mathrm{F}=$ estadístico de prueba. $\mathrm{p}=$ nivel de significación. En todos los casos p es menor a 0,05 por lo que indica la existencia de diferencias estadísticamente significativas.

Encontramos que dormir menos, acostarse tarde y de forma irregular, y reportar dificultades de sueño afecta al rendimiento académico de los niños.

Los niños que refieren niveles altos de estrés total y en las tres dimensiones, son los que pertenecen al grupo de rendimiento académico por debajo de la media, mientras que aquellos que menor percepción de estrés reportan, son los pertenecientes al grupo de mayor rendimiento académico.

Los estudiantes con un mejor perfil de sueño y menor estrés son los que obtienen un mejor rendimiento académico.

\section{CONCLUSIONES}

Los resultados muestran que los niños de primaria, en su mayoría, no duermen las horas recomendables para su desarrollo y refieren dificultades en la calidad del mismo. Estas horas de sueño perdidas no son recuperables, lo que, de forma acumulada es un factor que provoca que su rendimiento se vea afectado.

Un creciente cuerpo de investigaciones sugiere asociaciones recíprocas entre el sueño y la actividad del eje hipotalámico pituitario adrenocortical (HPA). En respuesta al estrés, el eje HPA se activa aumentando la liberación de cortisol en la circulación.

Encontramos investigaciones como la de Steiger (2002) quien indica fuertes asociaciones bidireccionales entre los efectos de la restricción del sueño en el funcionamiento del HPA, con aumentos en los niveles de cortisol después de la restricción del sueño. Así como las alteraciones en los patrones de sueño afectan el funcionamiento de HPA, alteracio- nes en HPA las hormonas también influyen en los patrones de sueño.

Los resultados de este estudio muestran como el desempeño de los niños depende de factores tanto escolares como familiares.

Encontramos que existen altos porcentajes de niños con preocupaciones motivadas por situaciones que les generan malestar en el ámbito familiar, escolar, de las relaciones con los iguales, o incluso del ámbito de la salud. Por ello, es necesario que los profesionales de la educación estemos alertas e informados ante algunos problemas de los estudiantes que pueden perturbar su rendimiento, como por ejemplo el estrés cotidiano.

Estos hallazgos muestran que esta problemática debe abordarse interdisciplinariamente desde la psicoeducación promover la salud de los escolares y optimizar sus desempeños.

Se han reportado asociaciones entre la disfunción del sueño y las dificultades de comportamiento, con la falta de atención y los trastornos atencionales. Beebe (2011) ha planteado que la falta de sueño causa, somnolencia, falta de atención y, deficiencias cognitivas y de comportamiento.

Es posible que una mayor calidad de sueño pueda ayudar a los niños a afrontar mejor los factores estresantes, como las actividades escolares, de manera más adecuada al poder controlar mejor su comportamiento. Un mejor descanso tendría un "efecto protector" para poder enfrentar mejor los desafíos de la vida diaria.

Comprender los mecanismos fisiológicos que están asociados con el sueño y el bienestar nos exige mayores esfuerzos de prevención e intervención para reducir ambos problemas, ya que como vemos estos se retroalimentan.

Los resultados obtenidos en el presente estudio deben interpretarse teniendo en cuenta las limitaciones de la autoevaluación a estas edades y una medida del rendimiento académico desde la percepción del docente y no a través de una prueba específica. Esto nos invita a seguir investigando y multiplicando los estudios en pro de conocer de forma más especifica el impacto de estas variables en esta franja etaria. 


\section{REFERENCIAS}

Astrobus J, Balter M, Battles J, Berger R, Bliwise D, Carskadon M, et al. (1993) Basics of sleep behavior. Los Angeles, CA: Sleep Research Society.

BaHamman, A., Al-Faris, E., Shaikh, S.H. y Bin Saeed, A. (2006). Sleep problems/habits and school performance in elementary school children. Sleep and Hypnosis, 8, 12-17.

Beebe, D.W. (2011) Cognitive, Behavioral, and Functional Consequences of Inadequate Sleep in Children and Adolescents Pediatric Clinics of North America Volume 58, Issue 3, Pages 649-665. DOI.org/10.1016/j.pcl.2011.03.002

Biggs, S.N., Lushington, K., Van den Heuvel, C.J., Martin, A.J., Kennedy, J.D. (2011). Inconsistent sleep schedules and daytime behavioral difficulties in school-aged children. Sleep Medicine, 12, 780-786

Brand, S., Gerber, M., Beck, J., Hatxinger, M.D., Pühse, U. y Holsboer-Trachsler, M.D. (2010). Journal of Adolescent Health, 46, 133-141.

Cladellas i Pros, R., Clariana, M., Gotzens Busquets, C., Badia Martín, M., \& Dezcallar Sáez, T. (2015). Patrones de descanso, actividades físico-deportivas extraescolares y rendimiento académico en niños y niñas de primaria. Revista de psicología del deporte, 24(1), 0053-59.

Del Río Portilla, I. Y. (2006) Estrés y sueño. Revista Mexicana de Neurociencia 7(1): 15-20.

Dewal, J. F., Meijer, A. M., Oort, F. J., Kerkhof, G. A. y Bögels, S. M. (2010). The influence of sleep quality, sleep duration and sleepiness on school performance

Dewal, J. F., Meijer, A. M., Oort, F. J., Kerkhof, G. A. y Bögels, S. M. (2010). The influence of sleep quality, sleep duration and sleepiness on school performance in children and adolescents: A meta-analytic review. Sleep Medicine Reviews, 14, 179-189.
Fallone, G., Acebo, C., Arnedt, J. T., Seifer, R., \& Carskadon, M. A. (2001). Effects of acute sleep restriction on behavior, sustained attention, and response inhibition in children. Perceptual and Motor Skills, 93, 213-229.

Fallone, G., Owens, J. A. y Deane, J. (2006). Sleepiness in children and adolescents: clinical applications. Sleep Medicine Reviews, 4, 287-306.

Feinberg I, Campbell IG. (2010) Sleep EEG changes during adolescence: an index of a fundamental brain reorganization. Brain Cogn; 72: 56-65.

González,M.J. Díaz-Giráldez,F.; Martin, I.; Delgado, M.; Trianes, M.V. (2014) Estrés cotidiano y precisión lectora en niños de educación primaria. Revista de Psicología, №1-Vol.3, pp:245-252 http://dx.doi.org/10.17060/ijodaep.2014.n1.v3.502.

Kanner, A.D., Coyne, J.C., Schaefer, C. y Lazarus, R.S. (1981). Comparison or two modes of stress measurement: Daily hassles and uplifts versus major life events. Journal of Behavioral Medicine, 4, 1-39.

Khalil, A.A. y Salah, N. (2012). The use of technology by university adolescent students and its relation to attention, sleep, and academic achievement Journal of American Science, 8(1), 264-270.

Lazarus, R. y Folkman, S. (1984). Stress, appraisal, and coping. Nueva York: Springer

López, M.; |Iglesia, F. (2019b). El costo del estrés diario en el rendimiento académico de niños. https://asociacioneducar.com/estresdiarioninos

López; M Iglesia, F. (2019a) Dormir poco y acostarse tarde afecta el rendimiento escolar. https://asociacioneducar.com/invdorre 


\section{REFERENCIAS}

Martínez-Vicente, M., Suárez-Riveiro, J. M., \& Valiente-Barroso, C. (2019). Estrés cotidiano infantil y factores ligados al aprendizaje escolar como predictores del rendimiento académico. Ansiedad y Estrés, 25(2), 111-117.

Maturana, H. A., \& Vargas, S. A. (2015). El estrés escolar. Revista Médica Clínica Las Condes, 26(1), 34-41.

Owens JA, Dalzell V (2005). Use of the 'BEARS' sleep detection tool in a continuity clinic for pediatric patients: a pilot study. Sleep Medicine 6 (1) 63-69.

Rosler, R.; Logatt Grabner, C.; Logatt Grabner, C.; Castro, M.; López, M.; Iglesia, F. (2019) "Sueño en niños argentinos de 6 a 12 años" XII Congreso Argentino de Neuropsicología. Sociedad de Neuropsicología de Argentina. Revista Argentina de Neuropsicología, 35, pág., 199 ISSN 1668 -5415.

Sandín, Bonifacio (2003). El estrés: un análisis basado en el papel de los factores sociales. International Journal of Clinical and Health Psychology [en linea]. 3(1), 141-157 ISSN: 1697-2600. Disponible en: https://www.redalyc.org/articulo.oa?id=33730109

Seiffge-Krenke, I. (2000). Causal links between stressful events, coping style and adolescent symptomatology. Journal of Adolescence, 23, 675- 691.

Selye, H. (1946). The general adaptation syndrome and the diseases of adaptation. The journal of clinical endocrinology, 6(2), 117-230.

Shellhaas RA, Burns JW, Hassan F, Carlson MD, Barks JDE, Chervin RD (2017). Neonatal Sleep-Wake Analyses Predict 18-month Neurodevelopmental Outcomes. Sleep; 40(11). doi: 10.1093/sleep/zsx144. 15.

Steiger, A. (2002) Sleep and the hypothalamo-pituitary-adrenocortical system. Sleep Medicine Reviews. DOI: org/10.1053/smrv.2001.0159.
Trianes Torres, M. V.; Fernández-Baena,F. J.; Escobar Espejo, M.; Blanca Mena, M. J.; co Maldonado Montero, E. . (2014) ¿Qué es el estrés cotidiano infantil?: Detección e intervención psicoeducativa. Revista Padres y Maestros. Núm. 360 Acción tutorial con las familias.

Trianes, M. V., Blanca, M. J., Fernández-Baena, F. J., Escobar, M. y Maldonado, E. F. (2011). IECl. Inventario de Estrés Cotidiano Infantil. Madrid: TEA Ediciones

Trianes, M. V., Blanca, M. J., Fernández-Baena, F. J., Escobar, M. y Maldonado. E. F. (2012). "Evaluación y tratamiento del estrés cotidiano en la infancia". Papeles del Psicólogo 32, 3-13.

Valiente-Barroso, C., Suárez-Riveiro, J. M., \& Martínez-Vicente, M. (2020). Rendimiento académico, aprendizaje y estrés en alumnado de primaria. Revista Complutense de Educación, 31(3), 365.

Velluti, R.A.; Pedemonte,M. (2006) Fisiología de la vigilia y el sueño en: Fisiología del cuerpo humano, Tresguerres J. A. F. Fisiologia humana. McGraw-Hill Interamericana.

Wagner, B.M., Compas, B.E., y Howell, D.C. (1988). Daily and major life events: A test of an integrative model of psychosocial stress. American Journal of Community Psychology, 16, 189-205

Yokomaku, A., Misao, K., Omoto, F., Yamagishi, R., Tanaka, K., Takada, K. y Kohyama, J. (2008). A study of the association between sleep habits and problematic behaviors in preschool children. Chronobiology International, 25(4), 549-564. 